\title{
Review
}

\section{How Mobile Health Technology and Electronic Health Records Will Change Care of Patients with Parkinson's Disease}

\author{
Clint Hansen ${ }^{\mathrm{a}}$, Alvaro Sanchez-Ferro ${ }^{\mathrm{b}}$ and Walter Maetzler ${ }^{\mathrm{a}, *}$ \\ ${ }^{a}$ Department of Neurology, Christian-Albrechts-Universität Kiel and University Hospital Schleswig-Holstein, \\ Campus Kiel, Kiel, Germany \\ ${ }^{\mathrm{b}}$ HM CINAC, Hospital Universitario HM Puerta del Sur, Universidad CEU-San Pablo, Móstoles, Madrid, Spain
}

\begin{abstract}
Care of patients with Parkinson's disease (PD) will dramatically change in the upcoming years. The nationwide implementations of the patient-controlled electronic health record (EHR) and the technology-based home monitoring system will most probably be the cornerstones of this revolution. We speculate that, within the course of the next decade, EHRs will lead to a substantial empowerment of patients, and monitoring of motor and non-motor manifestations of PD will shift from the clinic to the home. As far as this can be foreseen, small, partly clothing-embedded and implanted sensor systems allowing passive (i.e., non-obtrusive) data collection will dominate the market. They will interoperate with the personal EHR and other potentially health-related electronic databases such as clinical warehouses and population health analytics platforms. Analysis software will be mainly built on artificial intelligence, and presentation of data will be intuitive. This scenario will eventually help both the patient and the medical professional by providing higher amounts of quality information about daily-relevant effects of disease and treatment, eventually allowing for a better and more personalized care.
\end{abstract}

Keywords: Mobile health technology, electronic health records, Parkinson's disease, wearables

\section{ELECTRONIC HEALTH RECORDS AND MOBILE TECHNOLOGY: NEW PLAYERS WILL CHANGE PARKINSON'S DISEASE MANAGEMENT}

To date, digitization and technology are omnipresent in our societies and influence individual lives and human interaction almost everywhere and at any time. With broad support coming from governments of developed countries and the "big"

\footnotetext{
${ }^{*}$ Correspondence to: Prof. Dr. med. Walter Maetzler, ChristianAlbrechts-Universität zu Kiel, Klinik für Neurologie, UKSH, Campus Kiel, Arnold-Heller-Str. 3, Haus 41, 24105 Kiel, Germany. Tel.: +49 431500 23981; E-mail: w.maetzler@neurologie.unikiel.de.
}

technology players, this dynamic development is increasingly influencing, affecting and changing health care and medical decision-making. More specifically, this technological development has already led to (i) the implementation of electronic health records (EHRs) in a rapidly increasing number of countries and centers and (ii) a huge spectrum of technological and digital devices that can be worn on the body or placed in the home, allowing the detection of human function and behavior mainly in the usual environment. This viewpoint aims at presenting the current state and the potential future development of EHRs and technological devices with a focus on management of Parkinson's disease (PD). We argue that patients 
with PD can benefit from this development [1]. The disease requires significant engagement from both patients and medical professionals, making an interactive and flexible health data collection system such as an EHR particularly valuable for them. Moreover, the disease has many different motor features, and mobile health technology has advanced greatly in measuring movement and mobility and the evaluation of non-motor symptoms with apps and devices will gradually become possible. All these developments promise a continuous and daily life-relevant assessment of a broad spectrum of PD manifestations without being highly burdensome [2].

\section{ELECTRONIC HEALTH RECORDS}

Currently, most health records are paper-based and managed by medical institutions, which often have limited capacity to communicate with each other. Some health records have already migrated from paper charts to EHRs that are, however, largely digital remakes of the paper-based predecessors and do not integrate all the already available technical options that can support clinical decision making [3]. It is probable that this picture changes dramatically within the next decade. Most countries with established health structures will then have nationwide provision of innovative EHRs with cloud storage solutions, and most of these EHRs will be patient-controlled [4]. EHRs will communicate with clinical data warehouses that consolidate hospital- and practice-derived data, with population health analytics databases that will provide information about disease risk and prevention, and with digital technology that is used by the owner of the EHR, i.e., the patient [5] (Fig. 1). Actual policy decisions suggest that national health services will substantially support the empowerment of patients through technologies such as the EHR. Illustrative examples are the American Recovery and Reinvestment Act of 2009, spending 34 billion USD for the promotion and purchase of EHRs in the US [6], the Topol review commissioned by the Secretary of State for Health of the UK that has patient empowerment via the EHR in its focus [7], and the plan of the European Union to develop EHRs that allow patients to move with their EHRs flexibly across country borders [8].

We also anticipate that strong and far-reaching data protection rules and laws will reduce the risk of data leakage to a minimum and increase the acceptance rate of the EHRs among users. Professionals in the medical field will also benefit from the development as they will be able to focus more on the skills which initially made them to select their respective professions and which they have learned during their training [3].

\section{TECHNOLOGICAL AND DIGITAL DEVICES}

Digital technology, containing accelerometers, gyroscopes, magnetometers, heart rate monitors, galvanic skin resistors and barometers, will increasingly allow for continuous measurement of disease manifestations and resources in the usual environment of the user. Some of these, mainly mobile, devices are already available and widely accepted $[9,10]$, particularly when they are designed as a fashionable accessory such as a wristwatch, flexible form attached to the arms or feet, or integrated into the shoes [11, 12].

However, almost none of these devices are yet implemented in clinical routine, and none is used for clinical assessment of PD. Reasons may be that disease manifestations are complex and difficult to assess especially in the "uncontrolled" home environment, that most of the currently available feasibility and validity studies are small and not representative for different phases and subtypes of PD, that gold standards are difficult to define and often of limited accuracy, and that most of the business models of medical device companies are hindering a rapid and seamless (i.e., continuous) development and improvement of detection algorithms [13, 14]. These business models are built mainly on the problematic "build-and-freeze" rule, which means that software and algorithms are built, frozen, and then tested in unchanged form for its lifetime [15].

Still, we see a high potential of this communication and detection technology to change our care in chronic diseases, including PD. The abovementioned pitfalls and challenges are already, at least partly addressed by policy and research communities and roadmaps for adopting these technologies are currently developed.

We anticipate that, within the next decade, continuous monitoring systems will allow the collection of PD-relevant data across a broad spectrum of manifestations. They will most probably be easily and widely used and not requiring an active interaction with the user, will be approved by regulatory authori- 

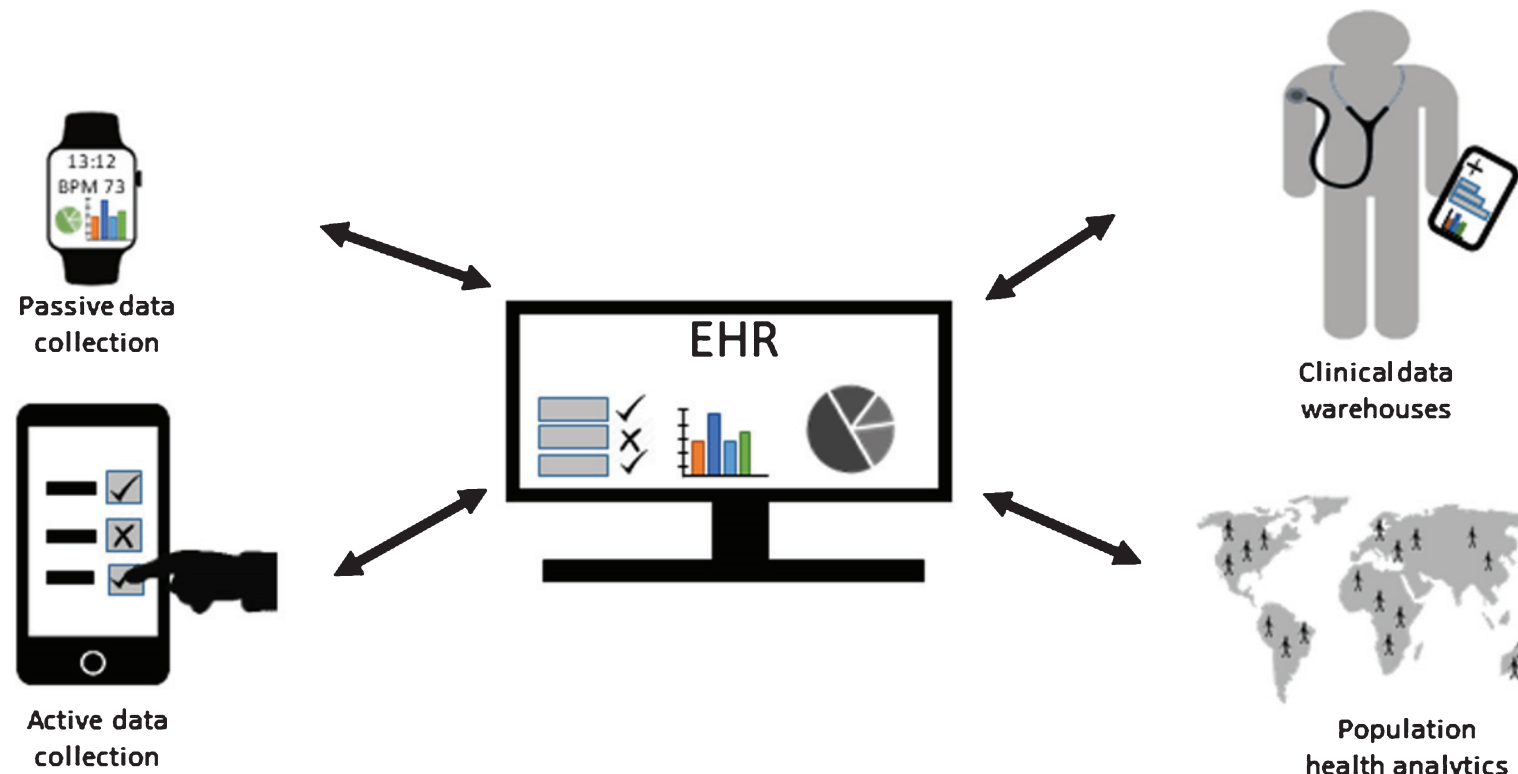

Clinical data

warehouses

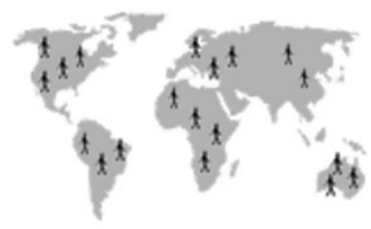

Population collection health analytics

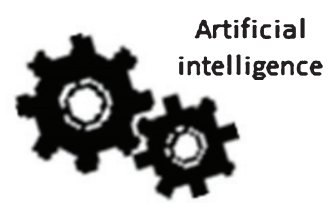

Fig. 1. The future of digital medicine will most probably be based on multiple interoperable electronic databases, with the patient-controlled electronic health record (EHR) in the center [4]. Passive and active data collection devices will be used by the patient and continuously feed data into the EHR (note that the watch in the figure stands for many different data collection opportunities, including non-body-located systems). Clinical data warehouses are institutional-located databases that will support medical professionals with clinical decision making and patient care in real time. Population health analytics will support health-related decision making by providing risk and prediction markers. Analysis strategies within and across databases will be mainly based on artificial intelligence algorithms. Adapted from http://www.neurologie.unikiel.de/en/neurogeriatrics/research.

ties and the costs will be covered by health insurances. The continuous subcutaneous measurement of blood glucose in diabetes patients can serve as a template for the development of similar devices within the Movement Disorders field [16]. Body function and performance testing systems can be very close to the body of the user without being continuously worn. An example is the unobtrusive measurement of the interaction of the patient with the environment, e.g., through keyboard typing (contact of the body with PC, smartphone speed of keyboard typing [17]). It is well imaginable that commands coming from speech and analyzed via sensors in hearing aids will become increasingly important in the near future. Hearing aids and glasses already have, or may soon have integrated detection systems. Moreover, earphones with integrated sensors that allow interaction with the environment and the user will soon be available ("closed-loop system"). At least some cognitive features may be assessed indirectly by analysis of mobility data, by analyzing reaction times, performances during multitasking and navigation performances including eye movement analysis. Devices may even be located in the body. Some DBS aggregates already contain accelerometers, and insertable cardiac monitors are good candidates for such a scenario. It is also probable that autonomic features, such as sweating and heart rate (variability), will be accurately quantified. Evaluation of gut motility is already possible with swallowed capsules that allow the evaluation of pressure, temperature, and $\mathrm{pH}$. This method will advance and smaller capsules will soon be available [18]. It is envisaged that at least the majority of these disease monitoring systems can be "individualized", i.e., they will be able to adapt to individual performance. For example, algorithms will adapt their accuracies for the assessment of hypokinesia, dyskinesia, on and off phenomena and fluctuations to each individual and replace the "one algorithm fits all approach". 
Many issues that currently hinder a smooth development of symptom-detecting devices in the field will be solved through an extensive evaluation of data derived from clinical trials that are including mobile device-extracted parameters as exploratory endpoints. This inclusive assessment battery strategy is strongly supported by the FDA and EMA. With this approach, the currently problematic and poorly understood limited comparability between the performance in a usual environment (where persons live) and the professional environment (where assessments may rather assess functional capacity aspects $[19,20])$ will be adequately addressed. Moreover, the "build-and-freeze" rule will be substituted by more seamless algorithm development approaches [15].

\section{THE INTERACTION BETWEEN ELECTRONICAL HEALTH RECORDS AND MOBILE TECHNOLOGY}

There is growing evidence that patients will be able to add data to their own EHR using different sources. These sources can be patient-owned "satellite" (mobile) technology coming from the health and fitness sector as well as medical digital systems as discussed above. It is anticipated that patients will be able to add data to or delete it from the EHR just as it can be on a smartphone, and there will be a marketplace for app and algorithm developers where they could compete with their third-party apps and sensor systems on aspects such as quality, price, value and user experience [6]. This scenario makes standardization of application programming interfaces (APIs) necessary. An API allows two independent digital systems to interact, and standardization allows to do that in predictable ways. EHRs from the US are already required to have such a standardized API [6]. Taken together, it is anticipated that within a few years, most PD patients will use an individual EHR and interoperable and substitutable technology that can interact with this EHR. This combination will allow the integration of daily-relevant data by use of algorithms that have still to be developed but promise the valid assessment of usual performance, with the eventual aim to improve knowledge about individual disease course and treatment response.

\section{CONCLUSION}

We anticipate a bright digital future for the monitoring of PD within the next decade. Patients can be substantially empowered by the collection, interpretation, and management of their own mobile health data. These data will, in combination with interoperable digital databases located in patients ' homes, hospitals, doctors' practices, and research institutions, enable personalized care at an entirely new level.

\section{CONFLICT OF INTEREST}

$\mathrm{CH}$ is working as a postdoc at the Neurology Department of the University Hospital SchleswigHolstein, Kiel. He has no conflicts of interest to report.

ASF research is sponsored by the "Consejería de Educación, Juventud y Deporte of Comunidad de Madrid" and the People Programme (Marie Curie Actions) of the European Union's Seventh Framework Programme (FP7/2007-2013) under REA agreement n 291820. He holds a patent on a Method and Apparatus for Motor Function Characterization and has received speaker honoraria from Teva, Zambon, Abbvie, Alter and Novartis Pharmaceuticals. Dr. Sánchez-Ferro is the current Chair of the Rating Scales Electronic Development Ad Hoc Committee.

WM receives or received funding from the European Union, The Michael J. Fox Foundation for Parkinson's Research, Robert Bosch Foundation, Neuroalliance, Lundbeck and Janssen, and holds part of a patent for the assessment of dyskinesias (German patent office, 102015220741.2). He received speaker honoraria from Abbvie, Bayer, UCB, Licher, and Rölke, and was invited to Advisory Boards of Market Access \& Pricing Strategy GmbH, Biogen and Abbvie. He serves as the Co-Chair of the Technology Task Force of the Movement Disorders Society.

\section{REFERENCES}

[1] van Uem JMT, Isaacs T, Lewin A, Bresolin E, Salkovic D, Espay AJ, Matthews H, Maetzler W (2016) A viewpoint on wearable technology-enabled measurement of wellbeing and health-related quality of life in Parkinson's disease. J Parkinsons Dis 6, 279-287.

[2] Sánchez-Ferro Á, Elshehabi M, Godinho C, Salkovic D, Hobert MA, Domingos J, van Uem JM, Ferreira JJ, Maetzler W (2016) New methods for the assessment of Parkinson's disease (2005 to 2015): A systematic review. Mov Disord 31, 1283-1292.

[3] Choi K, Gitelman Y, Asch DA (2018) Subscribing to your patients - reimagining the future of electronic health records. N Engl J Med 378, 1960-1962. 
[4] Mandl KD, Kohane IS (2016) Time for a patient-driven health information economy? N Engl J Med 374, 205-208.

[5] Althoff T, Sosič R, Hicks JL, King AC, Delp SL, Leskovec J (2017) Large-scale physical activity data reveal worldwide activity inequality. Nature 547, 336-339.

[6] Mandl KD, Kohane IS (2017) A 21st-century health IT system - Creating a real-world information economy. $N$ Engl J Med 376, 1905-1907.

[7] Health Education England (2018) The Topol Review: Preparing the healthcare workforce to deliver the digital future, interim report June 2018 - A call for evidence.

[8] COM (2018) 233 final: Communication from the Commission to the European Parliament, the Council, the European Economic and Social Committee and the Committee of the Regions on enabling the digital transformation of health and care in the Digital Single Mar, Last updated 2018, Accessed on 2018.

[9] Bernhard FP, Sartor J, Bettecken K, Hobert MA, Arnold C, Weber YG, Poli S, Margraf NG, Schlenstedt C, Hansen C, Maetzler W (2018) Wearables for gait and balance assessment in the neurological ward - study design and first results of a prospective cross-sectional feasibility study with 384 inpatients. BMC Neurol 18, 114.

[10] van Uem JMT, Maier KS, Hucker S, Scheck O, Hobert MA, Santos AT, Fagerbakke Ø, Larsen F, Ferreira JJ, Maetzler W (2016) Twelve-week sensor assessment in Parkinson's disease: Impact on quality of life. Mov Disord 31, 1337-1338.

[11] Lipsmeier F, Taylor KI, Kilchenmann T, Wolf D, Scotland A, Schjodt-Eriksen J, Cheng W-Y, Fernandez-Garcia I, Siebourg-Polster J, Jin L, Soto J, Verselis L, Boess F, Koller M, Grundman M, Monsch AU, Postuma RB, Ghosh A, Kremer T, Czech C, Gossens C, Lindemann M (2018) Evaluation of smartphone-based testing to generate exploratory outcome measures in a phase 1 Parkinson's disease clinical trial. Mov Disord 33, 1287-1297.
[12] Austen K (2015) What could derail the wearables revolution? Nat News 525, 22.

[13] Fröhlich H, Balling R, Beerenwinkel N, Kohlbacher O, Kumar S, Lengauer T, Maathuis MH, Moreau Y, Murphy SA, Przytycka TM, Rebhan M, Röst H, Schuppert A, Schwab M, Spang R, Stekhoven D, Sun J, Weber A, Ziemek D, Zupan B (2018) From hype to reality: Data science enabling personalized medicine. BMC Med 16, 150.

[14] Maetzler W, Klucken J, Horne M (2016) A clinical view on the development of technology-based tools in managing Parkinson's disease. Mov Disord 31, 1263-1271.

[15] Digital Health Software Precertification (Pre-Cert) Program, Last updated 2018, Accessed on 2018.

[16] Bolinder J, Antuna R, Geelhoed-Duijvestijn P, Kröger J, Weitgasser R (2016) Novel glucose-sensing technology and hypoglycaemia in type 1 diabetes: A multicentre, non-masked, randomised controlled trial. Lancet 388, 22542263.

[17] Giancardo L, Sánchez-Ferro A, Arroyo-Gallego T, Butterworth I, Mendoza CS, Montero P, Matarazzo M, Obeso JA, Gray ML, Estépar RSJ (2016) Computer keyboard interaction as an indicator of early Parkinson's disease. Sci Rep $\mathbf{6}$, 34468.

[18] Travers GJS, Nichols DS, Farooq A, Racinais S, Périard JD (2016) Validation of an ingestible temperature data logging and telemetry system during exercise in the heat. Temperature 3, 208-219.

[19] Artusi CA, Mishra M, Latimer P, Vizcarra JA, Lopiano L, Maetzler W, Merola A, Espay AJ (2018) Integration of technology-based outcome measures in clinical trials of Parkinson and other neurodegenerative diseases. Parkinsonism Relat Disord 46(Suppl 1), S53-S56.

[20] Giannouli E, Bock O, Mellone S, Zijlstra W (2016) Mobility in old age: Capacity is not performance. Biomed Res Int 2016, 3261567. 